\title{
Multi-Walled Carbon Nanotubes Intratracheally Instilled in Rat Lung
}

\author{
K. Yamamoto, ${ }^{*}$ E. Kobayashi, ${ }^{*}$ A. Ogami, ${ }^{* *}$ and Y. Morimoto** \\ * National Institute of Advanced Industrial Science and Technology (AIST), Tsukuba, Japan \\ ** University of Occupational and Environmental Health, Kitakyushu, Japan
}

Industrial applications of nano-carbon materials such as fullerenes and carbon nanotubes were reported in many fields recently. However, the toxicity of these nano-materials for the human was not clear, so the toxicity test of nano-carbon materials is important. As for the observation of biological specimen using transmission electron microscope (TEM), the high contrast observation of the biological specimen was important, and then, the tissue specimens were usually stained with the heavy elements such as $\mathrm{U}$ and $\mathrm{Pb}$ to increase the contrast of image. In the case of toxicity test of the nano-carbon materials, we must observe the biological tissue containing the nano-carbon materials. However, it was difficult to observe the stained biological specimen with the nano-carbon materials, because the contrast from nano-carbon materials was weak and below the background of the staining heavy elements. Furthermore, the high-resolution observation is necessary for the nano-carbon materials in the biological tissue. It is difficult to observe at the atomic scale resolution using the biological TEM with a high contrast objective lens. In these cases, the energy-filtering TEM (EFTEM) with high-resolution pole piece is useful. In this study, the toxicity tests of the multi-walled carbon nanotubes (MWCNTs) were performed using the intratracheally instilled in rat lung, and EFTEM observations of the rat lung were examined.

The in-vivo tests of MWCNTs were the intratracheal instillation of test material solution in the rat lung. Triton dispersions of $1 \mathrm{~g} / 1$ content were used for MWCNTs solution. The mounts of MWCNTs in the solutions were $200 \mu \mathrm{g}$ or $1 \mathrm{mg}$ for a body. The TEM images of MWCNTs in the test solution are shown in Figure 1(a) and (b). The diameter of MWCNTs is $50 \mathrm{~nm}$, and the length was ranging from 0.5 to $5 \mu \mathrm{m}$. The test solution was intratracheally instilled in rat lung. The lung tissues after three days, one week, one month, three, six, and twelve months from the instillation were observed by TEM. The lung tissue was fixed using glutaraldehyde and osmium tetroxide solution, and then dehydrated in ethanol, and embedded in epoxy resin. Ultrathin sections were cut on a diamond knife with microtomy. The staining condition of the tissue specimen was examined. The zero loss TEM imaging of the rat lung tissue with MWCNTs is performed by using an energy-filtering TEM with the objective lens for the high-resolution imaging (Zeiss, EM922HR). The acceleration voltage was $200 \mathrm{kV}$. The electron spectroscopic zero-loss images are obtained at a loss energy of $0 \mathrm{eV}$ with an energy window-width of $20 \mathrm{eV}$. Low magnification images were also examined using conventional biological TEM (Hitachi, H-7000).

TEM zero loss image of the alveolar macrophage after 3 months instillation exposure of MWCNTs solution of $1 \mathrm{mg}$ dose was shown in Figure 2(a). Fibrous structures were observed at the cytoplasm of macrophage, and it's clarified that this fibers are MWCNTs (Figure 2(b)). According to highresolution observations (Figure 3), MWCNTs keep the tube and the graphitic structures. This is because that graphite is chemically stable. Zero loss images of the alveolar cells after 3 months instillation exposure of MWCNTs was examined. Inflammation of the alveolar cells is observed and MWCNTs are also observed in the inflammation area. The inflammation of the alveolar cells is also observed in the lung of $0.2 \mathrm{mg}$ dose after 1 month instillation exposure, however the inflammation 
of the alveolar cells recover after 3 month instillation exposure. MWCNTs still are observed in the alveolar macrophage and the alveolar cells in lung of $1 \mathrm{mg}$ dose after 12 months instillation exposure. MWCNTs still keep clear graphitic structure.

This work was supported by New Energy and Industrial Technology Development Organization (NEDO) of Japan.
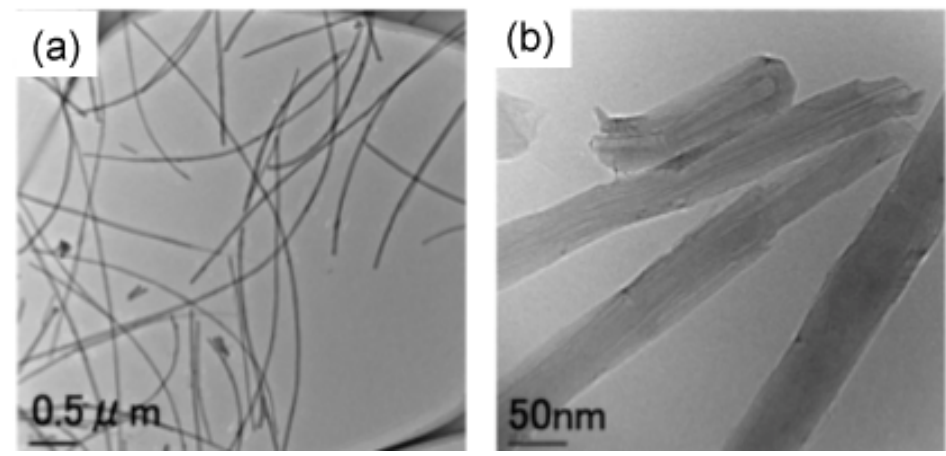

Figure 1. TEM photographs of MWCNTs in test solution (a). MWCNTs have graphitic tube structure (b).

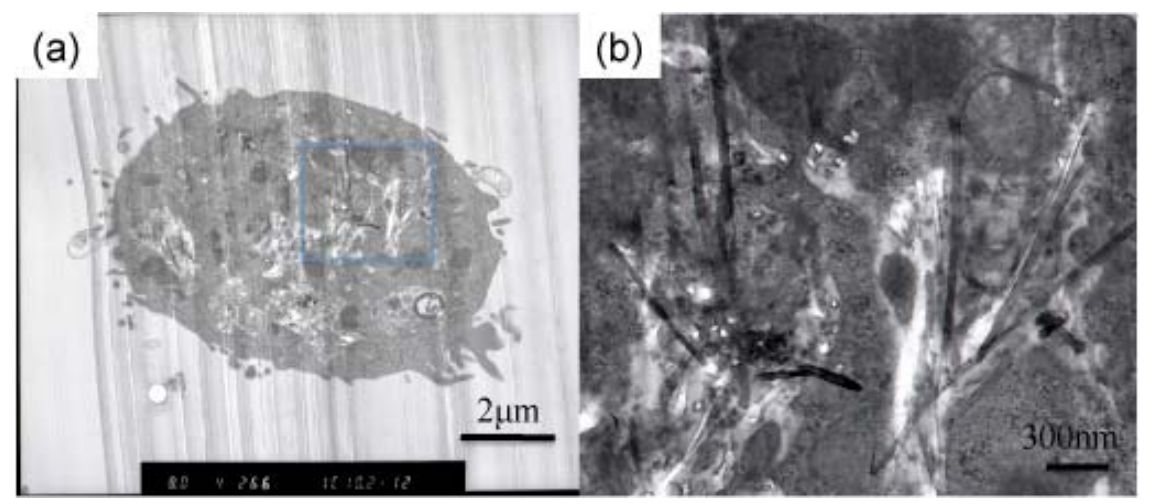

Figure 2. TEM images of alveolar macrophage after 3 months instillation exposure (a). (b) is magnified image of area in (a).

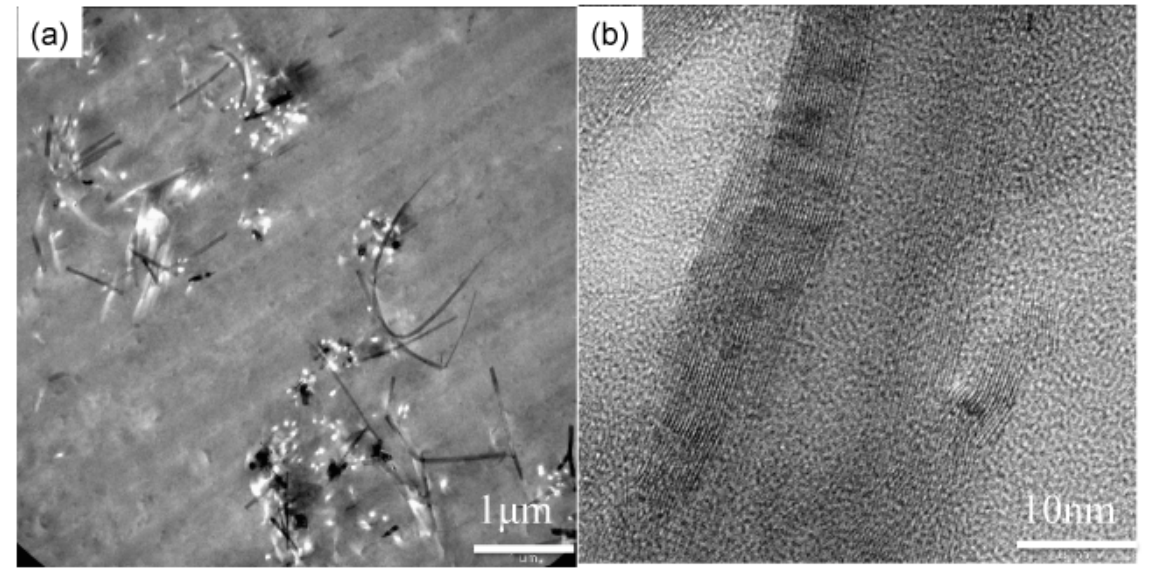

Figure 3. Zero loss TEM images of alveolar macrophage after 3 months instillation exposure (a). (b) is high resolution image of MWCNTs in (a). 